\title{
Slow gait speed - an indicator of lower cerebral vasoreactivity in type 2 diabetes mellitus
}

\author{
Azizah J. Jor'dan' ${ }^{1}$, Brad Manor ${ }^{1,2}$ and Vera Novak ${ }^{3}$ \\ 'Syncope and Falls in the Elderly Laboratory, Division of Gerontology, Department of Medicine, Beth Israel Deaconess Medical Center, \\ Harvard Medical School, Boston, MA, USA \\ 2 Institute for Aging Research, Hebrew SeniorLife, Harvard Medical School, Boston, MA, USA \\ ${ }^{3}$ Department of Neurology, Beth Israel Deaconess Medical Center, Harvard Medical School, Boston, MA, USA
}

\section{Edited by:}

Philip P. Foster, The University of Texas Health Science Center at Houston, USA

\section{Reviewed by:}

Richard Camicioli, McGill University, Canada

Franziska Matthäus, University of Heidelberg, Germany

\section{*Correspondence:}

Azizah J. Jor'dan, Syncope and Falls in the Elderly Laboratory, Division of Gerontology, Department of Medicine, Beth Israel Deaconess Medical Center, Harvard Medical School, 185 Pilgrim Road, Palmer 117, Boston, MA 02215, USA e-mail: ajjordan@bidmc.harvard.edu
Objective: Gait speed is an important predictor of health that is negatively affected by aging and type 2 diabetes. Diabetes has been linked to reduced vasoreactivity, i.e., the capacity to regulate cerebral blood flow in response to $\mathrm{CO}_{2}$ challenges. This study aimed to determine the relationship between cerebral vasoreactivity and gait speed in older adults with and without diabetes.

Research design and methods: We studied 61 adults with diabetes ( $65 \pm 8$ years) and 67 without diabetes (67 \pm 9 years) but with similar distribution of cardiovascular risk factors. Preferred gait speed was calculated from a $75 \mathrm{~m}$ walk. Global and regional perfusion, vasoreactivity and vasodilation reserve were measured using 3-D continuous arterial spin labeling MRI at 3 Tesla during normo-, hyper- and hypocapnia and normalized for end-tidal $\mathrm{CO}_{2}$.

Results: Diabetic participants had slower gait speed as compared to non-diabetic participants $(1.05 \pm 0.15 \mathrm{~m} / \mathrm{s}$ vs. $1.14 \pm 0.14 \mathrm{~m} / \mathrm{s}, p<0.001)$. Lower global vasoreactivity $\left(r^{2}\right.$ adj $\left.=0.13, p=0.007\right)$, or lower global vasodilation reserve $\left(r^{2}\right.$ adj $\left.=0.33, p<0.001\right)$, was associated with slower walking in the diabetic group independently of age, BMI and hematocrit concentration. For every $1 \mathrm{~mL} / 100 \mathrm{~g} / \mathrm{min} / \mathrm{mmHg}$ less vasodilation reserve, for example, gait speed was $0.05 \mathrm{~m} / \mathrm{s}$ slower. Similar relationships between vasodilation reserve and gait speed were also observed regionally within the cerebellum, frontal, temporal, parietal, and occipital lobes $\left(r^{2}\right.$ adj $\left.=0.27-0.33, p<0.0001\right)$. In contrast, vasoreactivity outcomes were not associated with walking speed in non-diabetic participants, despite similar vasoreactivity ranges across groups.

Conclusion: In the diabetic group only, lower global vasoreactivity was associated with slower walking speed. Slower walking in older diabetic adults may thus hallmark reduced vasomotor reserve and thus the inability to increase perfusion in response to greater metabolic demands during walking.

Keywords: diabetes, gait, vasoreactivity, vasomotor, metabolic

\section{INTRODUCTION}

Gait speed is predictive of mobility, morbidity, and mortality in older adults (Guralnik et al., 1995; Studenski et al., 2011). Vasoreactivity is an important cerebrovascular control mechanism used to maintain brain perfusion during increased metabolic demands (Bullock et al., 1985; Schroeder, 1988) such as walking, and can be clinically quantified by the vasodilation responses to hypercapnia (Low et al., 1999; Lavi et al., 2006). In healthy older adults, blood flow velocities in the middle cerebral artery territory, which supplies numerous brain regions involved in locomotor control, increased proportionally to walking speed (Novak et al., 2007). In a population-based study comprising community-dwelling older adults both with and without risk factors for falls (e.g., diabetes, stroke, use of walking aids, etc.), slower walkers exhibited lower vasoreactivity within the middle cerebral artery territory as measured by Transcranial Doppler ultrasound (Sorond et al., 2010).
Slowing of gait may thus reflect an early manifestation of underlying abnormalities in vasoreactivity and perfusion adaptation to the metabolic demands of walking. However, the relationship between brain vascular health and walking has not yet been established.

Type 2 diabetes accelerates brain aging (Biessels et al., 2002; Last et al., 2007) and has also been linked with microvascular disease and altered cerebral blood flow regulation (Allet et al., 2008; Várkuti et al., 2011) and vasoreactivity (Novak et al., 2011). Diabetes is associated with reduced gait speed and related functional decline (Volpato et al., 2010). In older adults, gait characteristics have been linked to gray matter atrophy and white matter hyperintensities (Rosano et al., 2007a,b; Callisaya et al., 2013). Moreover, gray matter atrophy appears to have a stronger effect on locomotor control in those with type 2 diabetes as compared those without, suggesting that the control of walking may be more dependent upon supraspinal control within this population (Manor et al., 
2012). This study therefore aimed to determine the relationship between vasoreactivity and gait speed in older adults with and without type 2 diabetes. We hypothesized that lower global and regional vasoreactivity would be associated with slower gait speed in older adults, particularly in those with type 2 diabetes.

\section{MATERIALS AND METHODS PARTICIPANTS}

This secondary analysis was completed on prospectively collected data from community-dwelling older adults originally recruited via local advertisement. We analyzed records from three completed projects spanning March 2003-July 2012: Cerebral vasoregulation in the elderly with stroke (March 2003-April 2005); Cerebral perfusion and cognitive decline in type 2 diabetes (January 2006-December 2009); and Cerebromicrovascular disease in elderly with diabetes (August 2009-July 2012). Grant numbers are provided in the study funding section.

Collectively, these three studies recruited 447 participants who signed informed consent (212 non-diabetics, 151 diabetics, 84 stroke). 213 participants (103 non-diabetics, 69 diabetics, 41 stroke) were excluded at that time for the following reasons: (1) ineligible after the screening visit $(n=117)$; (2) withdrew consent $(n=31)$; (3) lost to follow-up $(n=13)$; (4) study terminated $(n=52)$ for reasons related to exclusion criteria or other reasons such as lack of permission from primary care provider, no transcranial Doppler insonation window, unstable/untreated hypertension, high BMI, cerebral palsy, claustrophobia, atrial fibrillation, inappropriate behavior during screening, metal implant, abdominal pain due to kidney stone, or entered a nursing home.

For the present analysis, we excluded an additional 43 stroke records that met the exclusion criteria for the current analyses, 34 records that did not have complete datasets, and 29 records from subjects who completed more than one of the above-mentioned studies. In each of the latter cases, the most recent record was kept. Thus, records from a total of 128 subjects were included in the present analysis.

Participants were originally screened by medical history and physical, neurological, and laboratory examinations. Research protocols were conducted in accordance with the ethical standards of the Beth Israel Deaconess Medical Center (BIDMC) Clinical Research Center and all participants signed an informed consent, as approved by the Institutional Review board at BIDMC.

The diabetic group included men and women aged $50-85$ years with a physician diagnosis and treatment of type 2 diabetes mellitus with oral agents and/or combinations with insulin for at least one year. Diabetes treatments included insulin, oral glucosecontrol agents (sulfonylurea, second generation agents), their combinations and diet. Non-diabetic participants had no history of metabolic disorder and were recruited to match the age and gender characteristics of the diabetic group (Table 1).

Exclusion criteria for the current analysis were history of stroke, myocardial infarction, clinically significant arrhythmia or other cardiac disease, nephropathy, severe hypertension (i.e., systolic $\mathrm{BP}>200$, diastolic $\mathrm{BP}>110 \mathrm{~mm} \mathrm{Hg}$ or the use of three or more antihypertensive medications), seizure disorder, kidney or liver transplant, renal disease, any other neurological or systemic disorder (aside from peripheral neuropathy), and current recreational drug or alcohol abuse. MRI exclusion criteria were incompatible metal implants, pacemakers, arterial stents, claustrophobia and morbid obesity (i.e., BMI > 40).

\section{PROTOCOL}

Participants completed medical history, autonomic symptoms, and physical activity questionnaires. A study physician completed physical, neurological, and ophthalmologic examinations. None of the study participants had active foot ulcers during the study. A study nurse completed a fasting blood draw and recorded vital signs, anthropometric and adiposity measures. Participants also completed a comprehensive cognitive exam, autonomic testing, perfusion MRI of the brain and a gait assessment. For this study, we focused analyses on gait and MRI-based measures of cerebral perfusion and vasoreactivity.

\section{Walking test}

A 12-min walk was completed along a $75 \mathrm{~m}$ course on an $80 \mathrm{~m} \times 4 \mathrm{~m}$ indoor hallway. Participants were instructed to walk at preferred speed (i.e., a pace they deemed as comfortable or normal), which has excellent test-retest reliability, even in those with severe diabetic complications (Steffen et al., 2002; Manor et al., 2008). The time taken to complete each $75 \mathrm{~m}$ length and total distance were recorded. For the present analysis, we only examined data from the first hallway length (i.e., the first $75 \mathrm{~m}$ of the trial) in order to minimize potential confounders of turning and fatigue. Assistive devices were not used for ambulation. A rating of perceived exertion was asked of the participant before the start of the walk and once the walk was completed. Rating of perceived exertion ranged from 0 (no exertion) to 10 (very, very strong exertion).

\section{Magnetic resonance imaging (MRI)}

Brain imaging was completed in a 3T GE HDx MRI scanner (GE Medical Systems, Milwaukee, WI, USA) within the Center for Advanced MR Imaging at the BIDMC. 3D spiral continuous arterial spin labeling (CASL) MRI was used to quantify cerebral perfusion (Alsop and Detre, 1998; Detre et al., 1998; Floyd et al., 2003) during normocapnia, hypocapnia, and hypercapnia. Vasoreactivity was assessed as perfusion responses to vasodilation during hypercapnia and vasoconstriction to hypocapnia (Kety and Schmidt, 1948), as a noninvasive reliable method of assessing the integrity of cerebral vasculature (Fujishima etal., 1971; Yen etal., 2002). Specifically, two-minute scans were acquired during normal breathing (i.e., baseline normocapnia; end tidal $\mathrm{CO}_{2}$ concentration 33-38 mmHg), hyperventilation (i.e., hypocapnia; participants hyperventilated to reduce $\mathrm{CO}_{2}$ to a target of $25 \mathrm{mmHg}$ ), and rebreathing (i.e., hypercapnia; participants breathed a mixture of $5 \% \mathrm{CO}_{2}$ and $95 \%$ air to increase $\mathrm{CO}_{2}$ to a target of $45 \mathrm{mmHg}$ ).

Respiratory rate, tidal volume and end-tidal $\mathrm{CO}_{2}$ values were measured during each scan using an infrared end-tidal volume gas monitor (Capnomac Ultima, General Electric, Fairfield, CT, USA) attached to a face-mask. Blood pressure and heart rate were also recorded at one-minute intervals using an upper-arm automatic blood pressure cuff and finger photoplethysmogram. 
Table 1 | Demographic characteristics of the non-diabetic and diabetic groups.

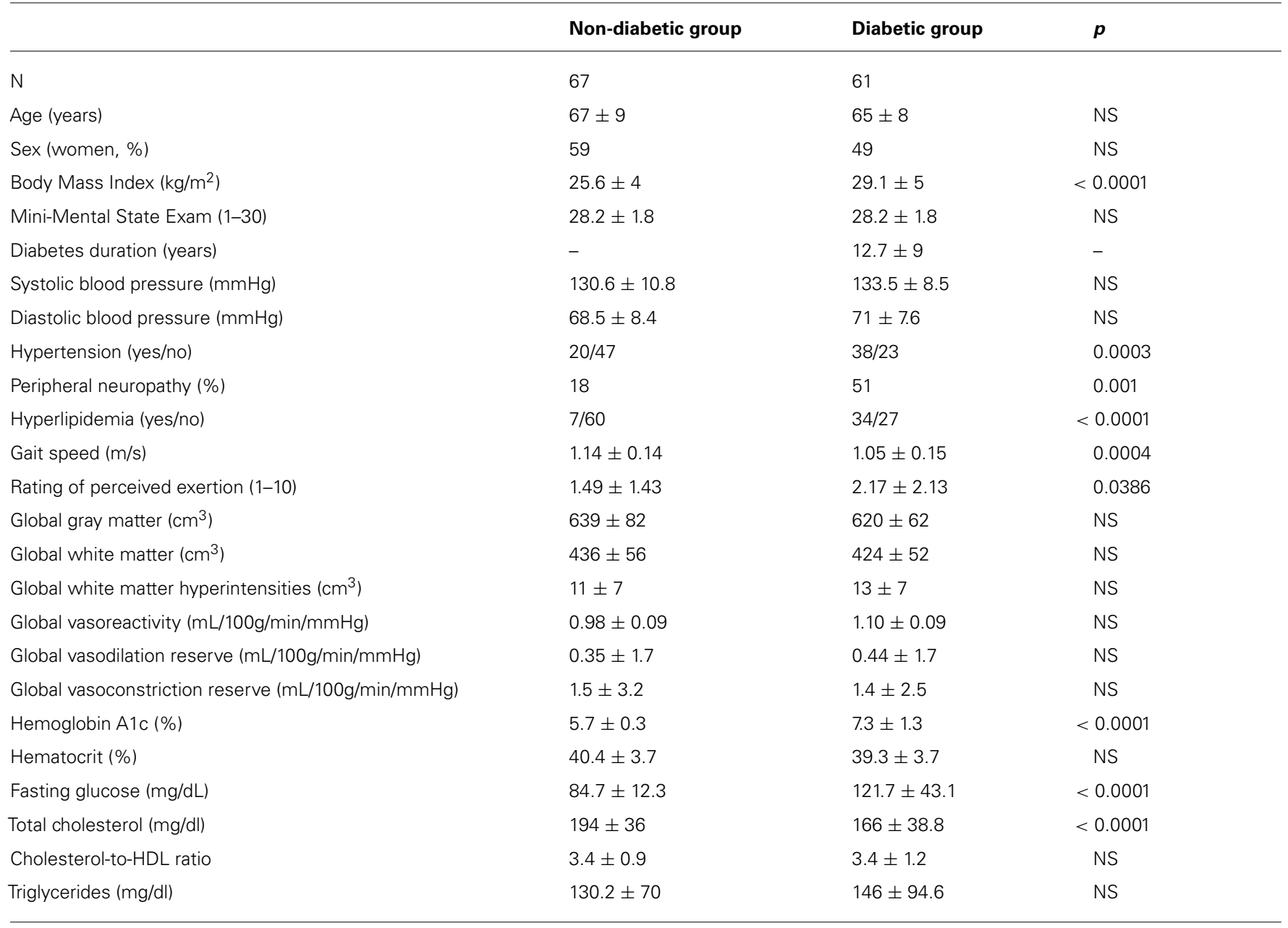

Data $=$ means $\pm S D$ unless otherwise indicated. $p=$ between-group comparisons. $N S=$ non-significant.

Perfusion images were acquired using a custom 3D CASL sequence $\left(T_{\mathrm{R}} / T_{\mathrm{E}}=10.476 / 2.46 \mathrm{~ms}\right.$, Label duration $=1.45 \mathrm{~s}$, postlabel delay $=1.525 \mathrm{~s}$, with $64 \times 64$ matrix in the axial plane and 40 slices with thickness $=4.5 \mathrm{~mm}$, seven spiral interleaves and the bandwidth $=125 \mathrm{kHz}$ ). Images were averaged over each condition to maximize signal-to-noise ratio.

A T1-weighted MP-RAGE structural imaging sequence was completed and used for registration of CASL images. Imaging parameters were: $\mathrm{TE} / \mathrm{TR}=3.3 / 8.1 \mathrm{~ms}$, flip angle of $10^{\circ}, 1-3 \mathrm{~mm}$ slice thickness, $24 \mathrm{~cm} \times 19 \mathrm{~cm}$ field of view (FOV), $256 \times 192$ matrix size.

\section{DATA ANALYSIS \\ Gait speed}

Average gait speed (m/s) was computed from the first $75 \mathrm{~m}$ of walking by dividing distance by time. This valid and reliable outcome predicts future health status and functional decline in numerous older adult populations (Quach et al., 2011; Studenski et al., 2011).

\section{Image analysis}

A rigid-body model (Collignon et al., 1995; Wells et al., 1996) was used for registration of the MP-RAGE image on CASL images using the Statistical Parametric Mapping software package (SPM, Wellcome Department of Imaging Neuroscience, University College, London, UK). This "normalization" module was employed to stereotactically normalize structural images to a standard space defined by ideal template image(s). The registered perfusion image was then overlaid on the segmented anatomical regions to obtain regional perfusion measurements. Generated maps of gray matter and white matter were segmented based upon the LONI Probabilistic Brain Atlas (Shattuck et al., 2008) and was used to calculate global volumes. All image segmentations were completed using Interactive Data Language (IDL, Research Systems, Boulder, CO, USA) and MATLAB (MathWorks, Natick, MA, USA) software.

\section{Perfusion analyses}

Perfusion and vasoreactivity were calculated in five regionsof-interest: the cerebellum, frontal, temporal, parietal, and occipital lobe. Within each region, perfusion was normalized for tissue volume and thus expressed in $\mathrm{mL} / 100 \mathrm{~g} / \mathrm{min}$. Four perfusion measures were calculated for each region: baseline perfusion during normal breathing, cerebral vasoreactivity, 
vasodilation reserve, and vasoconstriction reserve. Each outcome was computed globally and within each brain region-ofinterest.

Perfusion values were normalized to each subject's average $\mathrm{CO}_{2}$ level during this condition. Vasoreactivity measures were calculated as previously described (Last et al., 2007; Hajjar et al., 2010; Novak et al., 2011). Briefly, vasoreactivity was defined as the slope of the best-fit line produced by linear regression of perfusion and $\mathrm{CO}_{2}$ values across the three conditions (i.e., normal breathing, $\mathrm{CO}_{2}$ rebreathing, and hyperventilation). Vasodilation reserve was defined as the increase in perfusion from baseline to the rebreathing condition, normalized to the change in $\mathrm{CO}_{2}$ between these two conditions. Vasoconstriction reserve was defined as the decrease in perfusion from baseline to the hyperventilation condition, normalized to the change in $\mathrm{CO}_{2}$ between these two conditions.

\section{STATISTICAL ANALYSIS}

All analyses were performed using JMP software (SAS Institute, Cary, NC, USA). Descriptive statistics were used to summarize all variables. Outcomes have been expressed as either the mean $\pm \mathrm{SD}$ or categorical (yes/no) for each group. Student's $t$, Fisher's Exact and Chi-squared tests were used to compare group demographics.

We examined the effects of diabetes on both perfusion measures and gait speed using ANCOVA. For perfusion measures, the model effect was group and covariates included age, hematocrit (Hct) concentration and hypertension. Hct was included because it is inversely correlated with blood viscosity and is higher in men than women (Wells and Merrill, 1962; Kameneva et al., 1999; Zeng et al., 2000). Hypertension was included as a covariate because it affects small blood vessels of the body and may therefore alter cerebral blood flow regulation (Alexander, 1995; Hajjar et al., 2010). For gait speed, the model effect was group and covariates included age, gender and BMI.

Linear least-square regression analyses were used to test the hypotheses that (1) those with lower vasoreactivity demonstrate slower preferred gait speed, and (2) this association between vasoreactivity and gait speed is stronger (as reflected in the correlation coefficient, $r^{2}$ adj ) in older adults with diabetes as compared to those without diabetes. The dependent variable was gait speed. Model effects included perfusion outcome, group (non-diabetic, diabetic), and their interaction. Separate models were performed for each global and regional perfusion and vasoreactivity outcome. Age, BMI, and Hct concentration were included as covariates. Significance level was set to $p=0.05$ for each global perfusion and vasoreactivity outcome. The Bonferroni-adjusted significance level for multiple comparisons $(p=0.01)$ was used to determine significance of models examining outcomes within each of the five brain regions-of-interest.

\section{RESULTS}

\section{PARTICIPANTS}

Groups were matched by age and gender and had a similar cardiovascular risk factors (e.g., blood pressure, triglycerides, cardiovascular disease history), yet the diabetic group had higher BMI $(p<0.0001)$. The prevalence of hypertension and peripheral neuropathy was also higher in the diabetic group as compared to the non-diabetic group (62\% vs. $30 \%, p<0.001$ and $51 \%$ vs. $18 \%$, $p<0.001$, respectively). Participants with diabetes had greater $\mathrm{HbA1c}$ and serum glucose levels, but lower total cholesterol as compared to the non-diabetic group. Blood Hct concentration was similar between groups, but overall, higher in males as compared to females ( $42 \%$ vs. $38 \%, p<0.001$ ). Groups did not differ in global gray matter, white matter or white matter hyperintensity volumes (see Table 1).

\section{THE EFFECTS OF DIABETES ON PERFUSION AND CEREBRAL VASOREACTIVITY \\ Baseline perfusion and cerebral vasoreactivity}

The diabetic and non-diabetic groups had similar global and regional perfusion at baseline after normalizing for baseline $\mathrm{CO}_{2}$ levels and adjusting for age, Hct concentration and the presence of hypertension. Global and regional vasoreactivity, as well as vasodilation and vasoconstriction reserve, were also similar between groups (Table 1).

\section{THE EFFECTS OF DIABETES ON GAIT SPEED}

The diabetic group had slower preferred gait speed as compared to the non-diabetic group $(1.05 \pm 0.15 \mathrm{~m} / \mathrm{s}$ vs. $1.14 \pm 0.14 \mathrm{~m} / \mathrm{s}$, $p<0.001$; Table 1). This group difference remained significant $(p=0.007)$ after adjusting for age, gender, and BMI.

Across all participants, those with higher BMI had slower gait speed $\left(r^{2}\right.$ adj $\left.=0.04, p=0.01\right)$. Specifically, within the diabetic group, those with higher fasting glucose had slower gait speed $\left(r^{2}\right.$ adj $\left.=0.13, p=0.003\right)$. Gait speed was not correlated with the participant's rating of perceived exertion, HbAlc levels or diabetes diagnosis duration. The diabetic group had a higher change in rating of perceived exertion (i.e., difference from the start of walk from the end of the walk) compared to the non-diabetic group $(2.17 \pm 2.13$ vs. $1.49 \pm 1.43, p=0.039)$.

\section{RELATIONSHIPS BETWEEN CEREBRAL VASOREACTIVITY AND GAIT SPEED \\ Cerebral vasoreactivity}

Least square models revealed that global vasoreactivity was related to gait speed, but that this relationship was dependent upon group $\left(F_{1,96}=5.48, p=0.024\right)$. This group by vasoreactivity interaction was independent of age, BMI, and Hct levels. Post hoc testing indicated that within the diabetic group, those with lower global vasoreactivity walked more slowly $\left(r^{2}{ }_{\text {adj }}=0.13, p=0.007\right.$; Figures 1A,B). In the non-diabetic group, however, global vasoreactivity was not correlated with gait speed (Figure 1C). A trend towards a similar interaction was also observed between frontal lobe vasoreactivity and group $\left(F_{1,95}=4.32, p=0.04\right)$; that is, in the diabetic group only, those with lower frontal lobe vasoreactivity tended to walk slower $\left(r^{2}\right.$ adj $\left.=0.13, p=0.007\right)$. Yet, this interaction was not significant based upon the Bonferroni-adjusted significance level $(p=0.01)$.

\section{Vasodilation reserve}

Least square models revealed a significant relationship between global vasodilation reserve and gait speed, but that this relationship was also dependent upon group $\left(F_{1,97}=12\right.$, 
A Diabetic Participant 1: Gait Speed $=1.08 \mathrm{~m} / \mathrm{s}$; Global Vasoreactivity $=2.04$ *

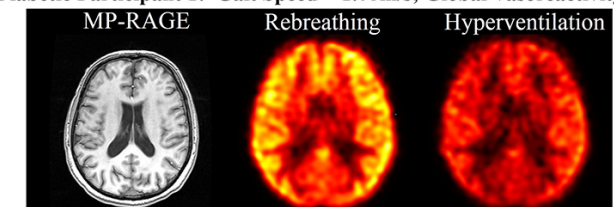

Diabetic Participant 2: Gait Speed $=0.82 \mathrm{~m} / \mathrm{s}$; Global Vasoreactivity $=1.29 *$

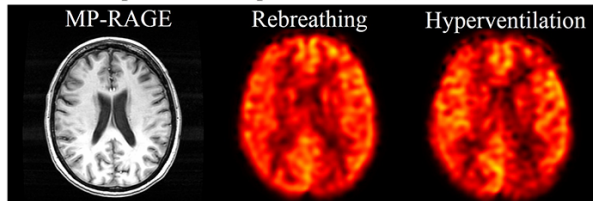

B

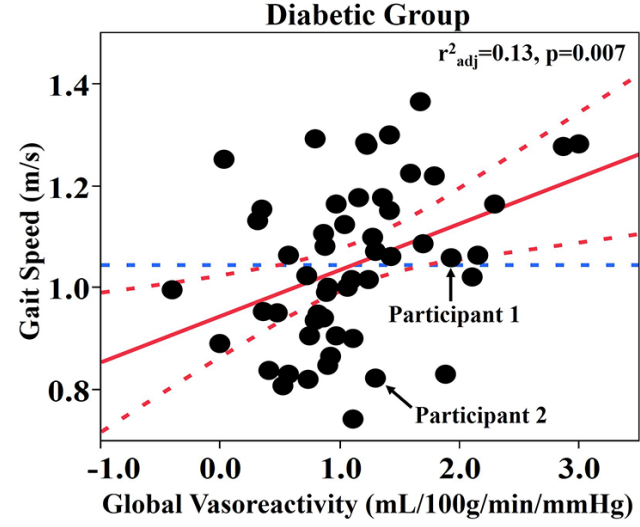

C

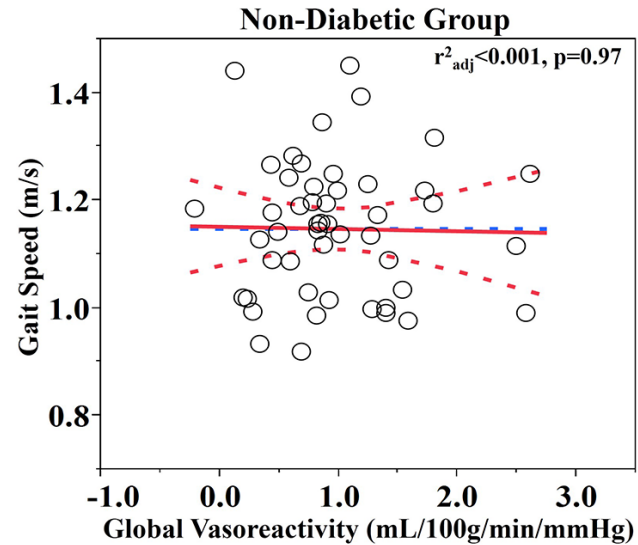

FIGURE 1 | (A) Reconstructed anatomical (i.e., MP-RAGE) and perfusion maps for two participants with type 2 diabetes mellitus. The top row represents a participant with diabetes that has high global vasoreactivity and fast gait speed (see Diabetic Participant 1 in $\mathbf{A}$ ). The bottom row represents a participant with diabetes that has low global vasoreactivity and slow gait speed (see Diabetic Participant 2 in A). (B) The relationship between global vasoreactivity and gait speed in the diabetic group. (C) The relationship between global vasoreactivity and gait speed in the non-diabetic group. Vasoreactivity was calculated as the change in perfusion from hypocapnia (hyperventilation) to hypercapnia $\left(\mathrm{CO}_{2}\right.$ rebreathing) conditions, normalized to the change in $\mathrm{CO}_{2}$ values. Best fit red solid line; Confidence Intervals - red dotted lines; Gait speed mean - blue dotted line; ${ }^{*}$ unit $-\mathrm{mL} / 100 \mathrm{~g} / \mathrm{min} / \mathrm{mmHg}$. $p<0.001)$. This significant interaction between group and vasodilation reserve was independent of age, BMI, and Hct levels. Post-hoc testing revealed that within the diabetic group only, those with lower global vasodilation reserve walked more slowly $\left(r^{2}{ }_{\text {adj }}=0.33, p<0.0001\right.$; Figure 2A).

Similar interactions were present between group and vasodilation reserve within each brain region-of-interest (cerebellum: $F_{1,94}=13, p<0.001$; frontal lobe: $F_{1,96}=8.49, p=0.005$; temporal lobe: $F_{1,96}=17.1, p<0.001$; parietal lobe: $F_{1,95}=8.72$, $p=0.004$; occipital lobe: $\left.F_{1,95}=8.99, p=0.004\right)$. In each case, within the diabetic group only, those with lower vasodilation reserve walked slower (Least square: $r^{2}$ adj $=0.27-0.33$, $p \leq 0.001$; Table 2). In the non-diabetic group, neither global nor regional vasodilation reserve was correlated with gait speed (Figure 2B).

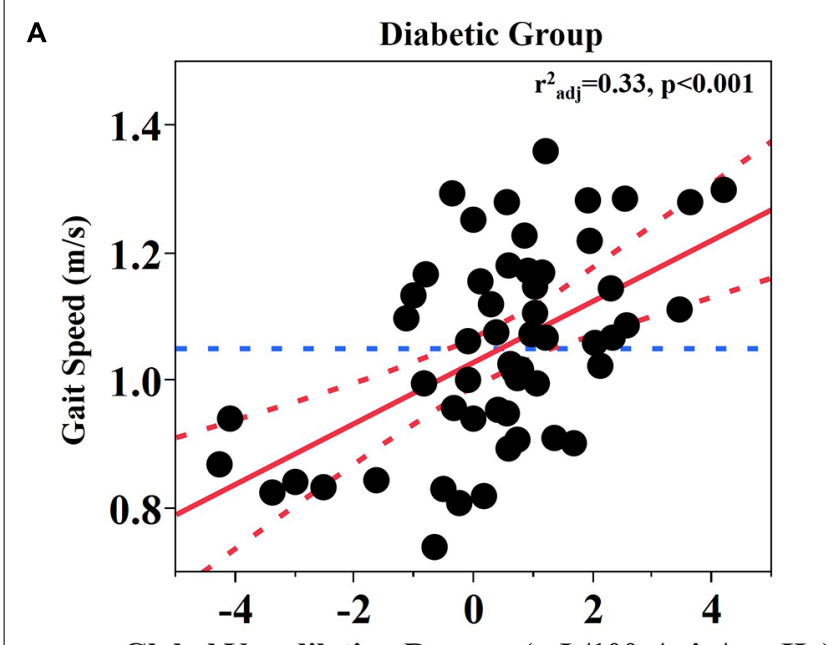

B

Global Vasodilation Reserve $(\mathrm{mL} / 100 \mathrm{~g} / \mathrm{min} / \mathrm{mmHg})$

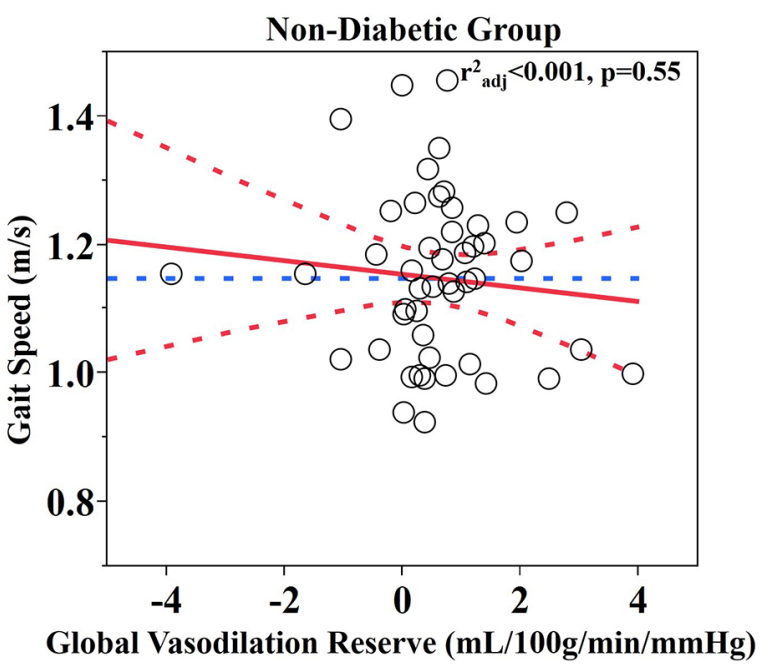

FIGURE 2 | Relationship between global vasodilation reserve and gait speed. (A) Diabetic group (B) Non-diabetic group. Best fit - red solid line; Confidence bounds - red dotted lines; Gait speed mean - blue dotted line. 
Table 2 | Vasodilation reserve and gait speed relationship in the diabetic group.

\begin{tabular}{|c|c|c|c|}
\hline & $M$ & $r^{2}$ adj & $\boldsymbol{p}$ \\
\hline Gait speed $(\mathrm{m} / \mathrm{s})$ Vasodilation reserve $(\mathrm{mL} / 100 \mathrm{~g} / \mathrm{min} / \mathrm{mmHg})$ & $1.05 \pm 0.02$ & & \\
\hline Global & $0.42 \pm 0.2$ & 0.33 & $<0.0001$ \\
\hline Cerebellum & $0.62 \pm 0.2$ & 0.33 & $<0.0001$ \\
\hline Frontal & $0.31 \pm 0.3$ & 0.27 & $<0.0001$ \\
\hline Temporal & $0.48 \pm 0.2$ & 0.33 & $<0.0001$ \\
\hline Parietal & $0.32 \pm 0.3$ & 0.30 & $<0.0001$ \\
\hline Occipital & $0.42 \pm 0.3$ & 0.29 & $<0.0001$ \\
\hline
\end{tabular}

Data are least square means $(M) \pm S E, r^{2}$ adj and $p$ value adjusted for age, BMI, and Hct.

\section{Vasoconstriction reserve}

Global and regional vasoconstriction was not related to gait speed in either group.

\section{Baseline perfusion}

Global or regional baseline perfusion was not related to gait speed within either group.

\section{Additional covariates}

Secondary analyses were performed to determine if within the diabetic group, the observed relationships between cerebral blood flow regulation outcomes and gait speed were influenced by the participant's height, weight, rating of perceived exertion, the burden of white matter hyperintensities, or the prevalence of hypertension or peripheral neuropathy. In each case, relationships between cerebral blood flow regulation and gait speed remained significant after adjusting for potential covariance associated with these factors.

\section{DISCUSSION}

This study has shown that within the diabetic group, those with lower global vasoreactivity walked more slowly. Our results further indicate that within this group, vasodilation reserve, or the capacity to increase cerebral perfusion specifically in response to hypercapnia, was linked to gait speed, which is an overall measure of health in older adults. This relationship was observed both globally and within each brain region-of-interest (i.e., cerebellum, frontal lobe, temporal lobe, parietal lobe, and occipital lobe). Specifically, for every $1 \mathrm{~mL} / 100 \mathrm{~g} / \mathrm{min} / \mathrm{mmHg}$ less global vasodilation reserve, gait speed was $0.05 \mathrm{~m} / \mathrm{s}$ slower in the diabetic group. These relationships were independent of age, BMI, Hct, and additional covariates (i.e., height, weight, rating of perceived exertion, white matter hyperintensities, and the prevalence of hypertension or peripheral neuropathy).

Both groups presented with average walking speeds that were slower than published norms; i.e., $1.2-1.4 \mathrm{~m} / \mathrm{s}$ for healthy adults over 50 years of age (Bohannon, 1997). Diabetic participants walked $0.09 \pm 0.15 \mathrm{~m} / \mathrm{s}$ more slowly than those without diabetes, which reflects a clinically significant difference between groups (Kwon et al., 2009). In the diabetic group, walking speed was correlated with fasting glucose levels, but not with diabetes duration or HbA1c. Furthermore, as can be observed in Figure 2A, several participants with diabetes that walked the slowest appeared to have abnormal responses to the hypercapnia condition (i.e., no change or decreased perfusion). For these individuals, this response may function as a compensatory response to ensure adequate perfusion even during resting conditions (Novak et al., 2006).

Previous research in older adults has linked slow gait speed to impaired "neurovascular coupling," or the change in cerebral blood flow in response to the performance of a cognitive task (Girouard and Iadecola, 2006; Iadecola and Nedergaard, 2007; Sorond et al., 2011). For example, Sorond et al. (2011) investigated the association between gait speed and neurovascular coupling as quantified by the change in blood flow velocity within the middle cerebral artery (using Transcranial Doppler Ultrasonography) in response to performance of the $n$-back cognitive task. Those with impaired neurovascular coupling walked more slowly. They also reported an interaction between neurovascular coupling and white matter hyperintensity burden, such that the presence of white matter hyperintensities was associated with reduced gait speed, except in those individuals with relatively strong neurovascular coupling. Previous work by Novak et al. (2007,2011) further demonstrated that lower vasoreactivity is linked to reduced gait speed independently of white matter hyperintensities specifically within older adults with type 2 diabetes. Therefore, neurovascular coupling appears to one mechanism that links vascular changes to neuronal activity, and is therefore essential for the preservation of functional outcomes. This notion is in line with the "brain reserve" hypothesis (Bullock et al., 1985; Stern, 2002) and may help explain the results of the current study. In other words, while diabetes was associated with reduced gait speed overall, those diabetic participants with greater vasoreactivity (or vasodilation reserve) tended to walk at similar speeds as non-diabetic controls.

Walking is a complex act that requires the coordination of locomotor, cardiovascular, and autonomic systems. The lack of relationship between cerebral vasoreactivity and gait speed in those without diabetes is supported by the notion that gait is largely autonomous and governed primarily by supraspinal elements of the motor control system under normal or healthy conditions (Stoffregen et al., 2000; Manor etal., 2010; Kloter et al., 2011). In those with diabetes, however, the capacity to 
modulate cerebral perfusion between conditions of hyper- and hypocapnia (i.e., vasoreactivity, a widely used prognosis of metabolic cerebral blood flow regulation) was associated with gait speed. These results suggest that in diabetic patients, the regulation of walking speed is dependent upon cerebral elements related to the locomotor control system. This notion is supported by research demonstrating that walking requires adjustments of the cardiovascular and cerebrovascular systems that are coordinated to increase blood pressure and cerebral blood flow velocities in order to meet metabolic demands (Novak et al., 2007; Perrey, 2013). Therefore, those diabetic participants with reduced vasoreactivity may have a diminished ability to increase perfusion in response to the metabolic demand associated with walking.

The relationship between vasoreactivity and gait speed that was observed in the diabetic group, but not in the non-diabetic group might also be explained by the complex effects of diabetes on cerebral vasculature and metabolism. Diabetes accelerates aging in the brain (Launer, 2006) and alters vascular reactivity through the combined effects of central insulin resistance on microvasculature, brain metabolism, glucose utilization, and neuronal survival. Central insulin plays an important role as a neuromodulator in key processes such as cognition (Shemesh et al., 2012; Freiherr et al., 2013), energy homeostasis, and glucose utilization during activity (e.g., walking). Cerebral insulin may directly modulate neuron-astrocyte signaling through neurovascular coupling and autonomic control of vascular tone and thus enable better regulation of local and regional perfusion (Lok et al., 2007) and neuronal activity in response to various stimuli (Amir and Shechter, 1987; Cranston et al., 1998; Kim et al., 2006; Muniyappa et al., 2007) including walking. Type 2 diabetes decreases insulin sensitivity in the brain, insulin transport through the blood-brain barrier, and insulin receptor's sensitivity, and it alters glucose metabolism and energy utilization (Plum et al., 2005, 2006; Hallschmid et al., 2007; Freiherr et al., 2013). Glucotoxicity and endothelial dysfunction associated with chronic hyperglycemia further affect perfusion, vasoreactivity, and metabolism (Makimattila and Yki-Jarvinen, 2002; Brownlee, 2005; Kilpatrick et al., 2010) and contribute to neuronal loss (Manschot et al., 2006, 2007; Last et al., 2007). Therefore, inadequate insulin delivery to brain tissue combined with altered energy metabolism may affect neuronal activity in multiple regions, but in particular the motor and cognitive networks that have high demands on energy (Gunning-Dixon and Raz, 2000). Diabetes may therefore especially alter neuronal activity and energy utilization during complex tasks like walking which require coordination of neuronal activity in numerous brain regions. As such, even if the same amount of blood flow is delivered to the neurons, energy utilization may be reduced in diabetic as compared to non-diabetic brain, leading to reduced neuronal activity and function, such as walking speed.

While our study controlled for numerous variables associated with gait speed, it did not control for other associated variables, such as muscular strength or fear of falling (Bendall et al., 1989; Chamberlin et al., 2005). The current study has the advantage of investigating regional perfusion in response to $\mathrm{CO}_{2}$ challenges using 3-D CASL MRI; however, the measures were recorded while participants were lying supine and not during walking. Although these regional perfusion measures may be lost, future studies are warranted to utilize wireless cerebral blood flow measurement tools (e.g., portable TCD or functional near-infrared spectroscopy) to examine the effects of diabetes on cerebral perfusion when walking at different speeds. Moreover, this is a cross-sectional study and thus, observed relationships between low vasoreactivity and slow gait speed does not necessarily imply a causal link between the two. As such, prospective studies are needed to determine potential mechanisms underlying the observed relationship between vasoreactivity and gait speed in those with diabetes, the predictive value of vasoreactivity as a clinical tool, and the potential for therapies targeting cerebral blood flow regulation to improve functional outcome in this vulnerable population.

\section{AUTHOR CONTRIBUTIONS}

Azizah J. Jor'dan analyzed the data, performed statistical analyses and wrote the manuscript. Brad Manor oversaw statistical analyses, data interpretation and contributed to manuscript preparation. Vera Novak designed the study, conducted experiments, and oversaw all aspects of the study, data interpretation and manuscript preparation.

\section{ACKNOWLEDGMENTS}

This work was conducted with support from a National Institute on Aging (NIA) T32 (5T32AG023480) fellowship awarded to Azizah J. Jor'dan, a KL2 Medical Research Investigator Training (MeRIT) award (1KL2RR025757-04) and NIA career development grant (1-K01-AG044543-01A1) awarded to Brad Manor, the Harvard Clinical and Translational Science Center (NIH Award KL2 RR 025757), and grants from the National Institute of Diabetes and Digestive and Kidney Diseases (5R21-DK-084463-02) and the NIA (1R01-AG-0287601-A2) awarded to Vera Novak. The content is solely the responsibility of the authors and does not necessarily represent the official views of Harvard Catalyst, Harvard University and its affiliated academic health care centers, the National Center for Research Resources, or the NIH.

\section{REFERENCES}

Alexander, R. W. (1995). Hypertension and the pathogenesis of atherosclerosis: oxidative stress and the mediation of arterial inflammatory response: a new perspective. Hypertension 25, 155-161. doi: 10.1161/01.hyp.25.2.155

Allet, L., Armand, S., Golay, A., Monnin, D., de Bie, R. A., and de Bruin, E. D. (2008). Gait characteristics of diabetic patients: a systematic review. Diabetes Metab. Res. Rev. 24, 173-191. doi: 10.1002/dmrr.809

Alsop, D. C., and Detre, J. A. (1998). Multisection cerebral blood flow MR imaging with continuous arterial spin labeling. Radiology 208, 410-416.

Amir, S., and Shechter, Y. (1987). Centrally mediated hypoglycemic effect of insulin: apparent involvement of specific insulin receptors. Brain Res. 418, 152-156. doi: 10.1016/0006-8993(87)90972-3

Bendall, M. J., Bassey, E. J., and Pearson, M. B. (1989). Factors affecting walking speed of elderly people. Age Ageing 18, 327-332. doi: 10.1093/ageing/18.5.327

Biessels, G. J., van der Heide, L. P., Kamal, A., Bleys, R. L., and Gispen, W. H. (2002). Ageing and diabetes: implications for brain function. Eur. J. Pharmacol. 441, 1-14. doi: 10.1016/S0014-2999(02)01486-3

Bohannon, R. W. (1997). Comfortable and maximum walking speed of adults age 20-79 years: reference values and determinants. Age Ageing 26, 15-19. doi: 10.1093/ageing/26.1.15 
Brownlee, M. (2005). The pathobiology of diabetic complications. Diabetes Metab. Res. Rev. 54, 441-452. doi: 10.2337/diabetes.54.6.1615

Bullock, R., Mendelow, A. D., Bone, I., Patterson, J., Macleod, W. N., and Allardice, G. (1985). Cerebral blood flow and $\mathrm{CO}_{2}$ responsiveness as an indicator of collateral reserve capacity in patients with carotid arterial disease. Br. J. Surg. 72, 348-351. doi: 10.1002/bjs.1800720506

Callisaya, M., Beare, R., Pham, T., Blizzard, L., Thrift, A. G., Chen, J., et al. (2013) Brain structural change and gait decline: a longitudinal population-based study. J. Am. Geriatr. Soc. 61, 1074-1079. doi: 10.1111/jgs.12331

Chamberlin, M. E., Fulwider B. D., Sanders, S. L., and Medeiros, J. M. (2005). Does fear of falling influence spatial and temporal gait parameters in elderly persons beyond changes associated with normal aging? J. Gerontol. A Biol. Sci. Med. Sci. 60, 1163-1167. doi: 10.1093/gerona/60.9.1163

Collignon, A., Maes, F., Delaere, D., Vancermeulen, D., Suetens, P., and Marchal, G. (1995). "Automated multi-modality image registration based on information theory," in Information Processing in Medical Imaging, eds Y. Bizais, C. Barillot, and R. Di Paola (Dordrecht: Kluwer Academic Publishers), 263-274.

Cranston, I., Marsden, P., Matyka, K., Evans, M., Lomas, J., Sonksen, P., et al. (1998). Regional differences in cerebral blood flow and glucose utilization in diabetic man: the effect of insulin. J. Cereb. Blood Flow Metab. 18, 130-140. doi: 10.1097/00004647-199802000-00002

Detre, J. A., Alsop, D. C., Vives, L. R., Maccotta, L., Teener, J. W., and Raps, E. C. (1998). Noninvasive MRI evaluation of cerebral blood flow in cerebrovascular disease. Neurology 50, 633-641. doi: 10.1212/wnl.50.3.633

Floyd, T. F., Ratcliffe, S. J., Wang, J., Resch, B., and Detre, J. A. (2003). Precision of the CASL-perfusion MRI technique for the measurement of cerebral blood flow with in whole brain and vascular territories. J. Magn. Reson. Imaging 18, 649-655. doi: 10.1002/jmri.10416

Freiherr, J., Hallschmid, M., Frey, W. H., Brunner, Y. F., Chapman C. D., Holscher, C., et al. (2013). Intranasal insulin as a treatment for Alzheimer's disease: a review of basic research and clinical evidence. CNS Drugs 27, 505-514. doi: 10.1007/s40263013-0076-8

Fujishima, M., Scheinberg, P., Busto, R., and Reinmuth, O. M. (1971). The relation between cerebral oxygen consumption and cerebral vascular reactivity to carbon dioxide. Stroke 2, 251-257. doi: 10.1161/01.str.2.3.251

Girouard, H., and Iadecola, C. (2006). Neurovascular coupling in the normal brain and in hypertension, stroke, and Alzheimer disease. J. Appl. Physiol. 100, 328-335. doi: 10.1152/japplphysiol.00966.2005

Gunning-Dixon, F. M., and Raz, N. (2000). The cognitive correlates of white matter abnormalities in normal aging: a quantitative review. Neuropsychology 14, 224 232. doi: 10.1037//0894-4105.14.2.224

Guralnik, J. M., Ferrucci, L., Simonsick, E. M., Salive, M. E., and Wallace, R. B. (1995). Lower-extremity function in persons over the age of 70 years as a predictor of subsequent disability. N. Engl. J. Med. 332, 556-561. doi: 10.1056/nejm199503023320902

Hajjar, I., Zhao, P., Alsop, D., and Novak, V. (2010). Hypertension and cerebral vasoreactivity: a continuous arterial spin labeling magnetic resonance imaging study. Hypertension 56, 859-864. doi: 10.1161/hypertensionaha.110. 160002

Hallschmid, M., Benedict, C., Born, J., and Kern, W. (2007). Targeting metabolic and cognitive pathways of the CNS by intranasal insulin administration. Expert Opin. Drug Deliv. 4, 319-322. doi: 10.1517/17425247.4.4.319

Iadecola, C., and Nedergaard, M. (2007). Glial regulation of the cerebral microvasculature. Nat. Neurosci. 10, 1369-1376. doi: 10.1038/nn2003

Kameneva, M. V., Watach, M. J., and Borovetz, H. S. (1999). Gender difference in rheologic properties of blood and risk of cardiovascular diseases. Clin. Hemorheol. Microcirc. 21, 357-363.

Kety, S. S., and Schmidt, C. F. (1948). The effects of altered arterial tensions of carbon dioxide and oxygen on cerebral blood flow and cerebral oxygen consumption of normal young men. J. Clin. Investig. 27, 484-492. doi: 10.1172/ jcil01995

Kilpatrick, E. S., Rigby, A. S., and Atkin, S. L. (2010). For debate. Glucose variability and diabetes complication risk: we need to know the answer. Diabet. Med. 27, 868-871. doi: 10.1111/j.1464-5491.2010.02929.x

Kim, J. A., Montagnani, M., Koh, K. K., and Quon, M. J. (2006). Reciprocal relationships between insulin resistance and endothelial dysfunction: molecular and pathophysiological mechanisms. Circulation 113, 1888-1904. doi: 10.1161/circulationaha.105.563213
Kloter, E., Wirz, M., and Dietz, V. (2011). Locomotion in stroke subjects: interactions between unaffected an affected sides. Brain 134, 721-731. doi: 10.1093/brain/awq370

Kwon, S., Perera, S., Pahor, M., Katula, J. A., King, A. C., Groessl, E. J., et al. (2009). What is a meaningful change in physical performance? Findings from a clinical trial in older adults (the LIFE-P study). J. Nutr. Health Aging 13, 538-544. doi: 10.1007/s12603-009-0104-Z

Last, D., Alsop, D. C., Abduljalil, A. M., Marquis, R. P., de Bazelaire, C., Hu, K., et al. (2007). Global and regional effects of type 2 diabetes on brain tissue volumes and cerebral vasoreactivity. Diabetes Care 30, 1193-1199. doi: 10.2337/ dc06-2052

Launer, L. J. (2006). Diabetes and brain aging: epidemiologic evidence. Curr. Diab. Rep. 5, 59-63. doi: 10.1007/s11892-005-0069-61

Lavi, S., Gaitini, D., Milloul, V., and Jacob, G. (2006). Impaired cerebral $\mathrm{CO}_{2}$ vasoreactivity: association with endothelial dysfunction. Am. J. Physiol. Heart Circ. Physiol. 291, H1856-H1861. doi: 10.1152/ajpheart.00014.2006

Lok, J., Gupta, P., Guo, S., Kim, W. J., Whalen, M. J., van Leyen, K., et al. (2007). Cell-cell signaling in the neurovascular unit. Neurochem. Res. 32, 2032-2045. doi: 10.1007/s11064-007-9342-9

Low, P. A., Novak, V., Spies, J. M., Novak, P., and Petty, G. W. (1999). Cerebrovascular regulation in the postural orthostatic tachycardia syndrome (POTS). Am. J. Med. Sci. 317, 124-133. doi: 10.1097/00000441-199902000-00007

Makimattila, S., and Yki-Jarvinen, H. (2002). Endothelial dysfunction in human diabetes. Curr. Diab. Rep. 2, 26-36. doi: 10.1007/s11892-0020054-X

Manor, B., Doherty, A., and Li, L. (2008). The reliability of physical performance measures in peripheral neuropathy. Gait Posture 28, 343-346. doi: 10.1016/j.gaitpost.2008.01.004

Manor, B., Hu, K., Zhao, P., Selim, M., Alsop, D., Novak, P., et al. (2010). Altered control of postural sway following cerebral infarction: a cross-sectional analysis. Neurology 76, 458-464. doi: 10.1212/WNL.0b013e3181cef647

Manor, B., Newton, E., Abduljalil, A., and Novak, V. (2012). The relationship between brain volume and walking outcomes in older adults with and without diabetic peripheral neuropathy. Diabetes Care 35, 1907-1912. doi: 10.2337/dc112463

Manschot, S. M., Biessels, G. J., de Valk, H., Algra, A., Rutten, G. E., van der Grond, J., et al. (2007). Metabolic and vascular determinants of impaired cognitive performance and abnormalities on brain magnetic resonance imaging in patients with type 2 diabetes. Diabetologia 50, 2388-2397. doi: 10.1007/s00125-007-0792-z

Manschot, S. M., Brands, A. M., van der Grond, J., Kessels, R. P., Algra, A., Kappelle, L. J., et al. (2006). Brain magnetic resonance imaging correlates of impaired cognition in patients with type 2 diabetes. Diabetes Metab. Res. Rev. 55, 11061113. doi: 10.2337/diabetes.55.04.06.db05-1323

Muniyappa, R., Montagnani, M., Koh, K. K., and Quon, M. J. (2007). Cardiovascular actions of insulin. Endocr. Rev. 28, 463-491. doi: 10.1210/er.2007-0006

Novak, V., Hu, K., Vyas, M., and Lipsitz, L. A. (2007). Cardiolocomotor coupling in young and elderly people. J. Gerontol. A Biol. Sci. Med. Sci. 62, 86-92. doi: 10.1093/gerona/62.1.86

Novak, V., Last, D., Alsop, D., Abduljalil, A. M., Hu, K., Lepicovsky, L., et al. (2006). Cerebral blood flow velocity and periventricular white matter hyperintensities in type 2 diabetes. Diabetes Care 29, 1529-1534. doi: 10.2337/ dc06-0261

Novak, V., Zhao, P., Manor, B., Sejdic, E., Alsop, D., Abduljalil, A., et al. (2011). Adhesion molecules, altered vasoreactivity, and brain atrophy in type 2 diabetes. Diabetes Care 34, 2438-2441. doi: 10.2337/dc11-0969

Perrey, S. (2013). Promoting motor function by exercising the brain. Brain Sci. 3, 101-122. doi: 10.3390/brainsci3010101

Plum, L., Belgardt, B. F., and Bruning, J. C. (2006). Central insulin action in energy and glucose homeostasis. J. Clin. Invest. 116, 1761-1766. doi: 10.1172/ jci29063

Plum, L., Schubert, M., and Bruning, J. C. (2005). The role of insulin receptor signaling in the brain. Trends Endocrinol. Metab. 16, 59-65. doi: 10.1016/j.tem.2005.01.008

Quach, L., Galica, A. M., Jones, R. N., Procter-Gray, E., Manor, B., Hannan, M. T., et al. (2011). The nonlinear relationship between gait speed and falls: the maintenance of balance, independent living, intellect, and zest in the elderly of Boston study. J. Am. Geriatr. Soc. 59, 1069-1073. doi: 10.1111/j.1532-5415.2011. 03408.x 
Rosano, C., Aizenstein, H. J., Studenski, S., and Newman, A. B. (2007a). A regions-of-interest volumetric analysis of mobility limitations in communitydwelling older adults. J. Gerontol. A Biol. Sci. Med. Sci. 62, 1048-1055. doi: 10.1093/gerona/62.9.1048

Rosano, C., Brach, J., Studenski, S., Longstreth, W. T. Jr., and Newman, A. B. (2007b). Gait variability is associated with subclinical brain vascular abnormalities in high-functioning older adults. Neuroepidemiology 29, 193-200. doi: $10.1159 / 000111582$

Schroeder, T. (1988). Hemodynamic significance of internal carotid artery disease. Acta Neurol. Scand. 77, 353-372. doi: 10.1111/j.1600-0404.1988.tb05921.x

Shattuck, D. W., Mirza, M., Adisetivo, V., Hojatkashani, C., Salamon, G., Narr, K. L., et al. (2008). Construction of a 3D probabilistic atlas of human cortical structures. Neuroimage 39, 1064-1080. doi: 10.1016/j.neuroimage.2007.09.031

Shemesh, E., Rudich, A., Harman-Boehm, I., and Cukierman-Yaffe, T. (2012). Effect of intranasal insulin on cognitive function: a systematic review. J. Clin. Endocrinol. Metab. 97, 366-376. doi: 10.1210/jc.2011-1802

Sorond, F. A., Galica, A., Serrador, J. M., Kiely, D. K., Iloputaife, I., Cupples, L. A., etal. (2010). Cerebrovascular hemodynamics, gait, and falls in an elderly population. Neurology 74, 1627-1633. doi: 10.1212/WNL.0b013e31 81df0982

Sorond, F. A., Kiely, D. K., Galica, A., Moscufo, N., Serrador, J. M., Ikoputaife, I. et al. (2011). Neurovascular coupling is impaired in slow walkers: the mobilize Boston study. Ann. Neurol. 70, 213-220. doi: 10.1002/ana.22433

Steffen, T. M., Hacker, T. A., and Mollinger, L. (2002). Age- and gender-related test performance in community-dwelling elderly people: Six-Minute Walk Test, Berg Balance Scale, Timed Up \& Go, and gait speeds. Phys. Ther. 82, 128-137.

Stern, Y. (2002). What is cognitive reserve? Theory and research application of the reserve concept. J. Int. Neuropsychol. Soc. 8, 448-460. doi: $10.1017 /$ S1355617702813248

Stoffregen, T. A., Pagulayan, R. J., Bardy, B. G., and Hettinger, L. J. (2000). Modulating postural control to facilitate visual performance. Hum. Mov. Sci. 19, 22-30. doi: 10.1016/s0167-9457(00)00009-9

Studenski, S., Perera, S., Patel, K., Rosano, C., Faulkner, K., Inzitari, M., et al. (2011). Gait speed and survival in older adults. J. Am. Med. Assoc. 305, 50-58. doi 10.1001/jama.2010.1923
Várkuti, B., Cavusoglu, M., Kullik, A., Schiffler, R., Yilmaz, O., Rosenstiel, W., et al. (2011). Quantifying the link between anatomical connectivity, gray matter volume and regional cerebral blood flow: an integrative MRI study. PLoS ONE 6:e14801. doi: 10.1371/journal.pone.0014801

Volpato, S., Maraldi, C., and Renato, F. (2010). Type 2 diabetes and risk for functional decline and disability in older persons. Curr. Diabetes Rev. 6, 134-143. doi: $10.2174 / 157339910791162961$

Wells, R. E., and Merrill, E. W. (1962). Influence of flow properties of blood upon viscosity-hematocrit relationships. J. Clin. Invest. 41, 159-1598. doi: 10.1172/JCI104617

Wells, W. M., Viola, P., Atsumi, H., Nakajima, S., and Kikinis, R. (1996). Multi-modal volume registration by maximization of mutual information. Med. Image Anal. 1, 35-51. doi: 10.1016/S1361-8415(01)80004-9

Yen, Y. F., Field, A. S., and Martin, E. M. (2002). Test-retest reproducibility of quantitative CBF measurements using FAIR perfusion MRI and acetazolamide challenge. Magn. Reson. Med. 47, 921-928. doi: 10.1002/mrm.10140

Zeng, S. M., Yankowitz, J., Widness, J. A., and Strauss, R. G. (2000). Etiology of differences in hematocrit between males and females: sequence-based polymorphisms in erythropoietin and its receptor. J. Gend. Specif. Med. 4, 35-40.

Conflict of Interest Statement: The authors declare that the research was conducted in the absence of any commercial or financial relationships that could be construed as a potential conflict of interest.

Received: 07 April 2014; accepted: 09 June 2014; published online: 26 June 2014. Citation: Jor'dan AJ, Manor B and Novak V (2014) Slow gait speed - an indicator of lower cerebral vasoreactivity in type 2 diabetes mellitus. Front. Aging Neurosci. 6:135. doi: 10.3389/fnagi.2014.00135

This article was submitted to the journal Frontiers in Aging Neuroscience.

Copyright (C) 2014 Jor'dan, Manor and Novak. This is an open-access article distributed under the terms of the Creative Commons Attribution License (CC BY). The use, distribution or reproduction in other forums is permitted, provided the original author(s) or licensor are credited and that the original publication in this journal is cited, in accordance with accepted academic practice. No use, distribution or reproduction is permitted which does not comply with these terms. 